\title{
The First Occurrence of Large Gaps Between Successive Primes
}

\author{
By Richard P. Brent
}

\begin{abstract}
A table of the first occurrence of a string of $2 r-1$ composite numbers between two primes is given for $r=158(1) 267,269,270,273,275,276,281,282,291,294$ and 301. All such strings between primes less than $2.6 \times 10^{12}$ have been accounted for. The computation supports some conjectures on the distribution of these strings.
\end{abstract}

1. Introduction. Let $p_{1}=2, p_{2}=3, \cdots$ be the sequence of primes. For positive integers $r$, define

$$
\begin{aligned}
f(r) & =p_{i} \quad \text { if } j \text { is the least positive index such that } p_{i+1}-p_{i}=2 r \\
& =\infty \text { if no such } j \text { exists, }
\end{aligned}
$$

and

$g(r)=p_{k} \quad$ if $k$ is the least positive index such that $p_{k+1}-p_{k} \geqq 2 r$.

Very little is known about the functions $f$ and $g$. It has not even been established that $f(r)$ is finite for all $r \geqq 1$. Certainly,

$$
F(r) \geqq G(r) \geqq c_{1} \log r \text { and } G(r) \leqq c_{2} r
$$

for positive constants $c_{1}$ and $c_{2}$, where $F(r)=\log f(r)$ and $G(r)=\log g(r)$. For these and other known results, see Johnson [8], Prachar [12], and Shanks [13].

Although the rigorous results are weak, heuristic probability arguments and empirical evidence suggest some plausible conjectures; see [1], [3]-[7], [10], [11], [13], and [15]. We give some empirical evidence which suggests that the function

$$
\phi(r)=\frac{F(r)-(2 r)^{1 / 2}}{\log (2 r)}
$$

is bounded, which implies that $\left|F(r)-(2 r)^{1 / 2}\right|$ and $\left|G(r)-(2 r)^{1 / 2}\right|$ are $O(\log r)$ as $r \rightarrow \infty$.

Lander and Parkin [10] have computed $f(r)$ for all values of $r$ such that $f(r) \leqq$ $1.096 \times 10^{10}$. In Section 2, we describe an extension of their computations. Since $g(r)=\min _{s \geqq r} f(s)$, it is sufficient to tabulate $f(r)$. Table 1 gives $f(r)$ for all $r \geqq 158$ such that $f(r) \leqq 2.6 \times 10^{12}$ (for $r=1, \cdots, 157$ see [10], where the notation $D=2 r$ and $P_{a}=f(r)$ is used). In Table 1 the "maximal" gaps (i.e., those for which $f(r)=g(r)$ ) are marked with an asterisk.

Received September 18, 1972.

AMS (MOS) subject classifications (1970). Primary 10-04, 10A20, 10A25, 10A40, 65A05.

Key words and phrases. Prime, distribution of primes, prime gap, maximal prime gap, successive composites, consecutive primes. 
TABLE 1

The First Occurrence of $2 r-1$ Consecutive Composite Numbers

\begin{tabular}{|c|c|c|c|}
\hline$r$ & $f(r)$ & $r$ & $f(r)$ \\
\hline 158 & 12109172293 & 198 & 50806025873 \\
\hline 159 & 4372999721 & 199 & 40267027589 \\
\hline $160 *$ & 2300942549 & 200 & 47203303159 \\
\hline 161 & 7961074441 & 201 & 44293346177 \\
\hline 162 & 10958687879 & 202 & 144999022043 \\
\hline 163 & 5837935373 & 203 & 49306638307 \\
\hline 164 & 13086861181 & 204 & 134664608389 \\
\hline 165 & 6291356009 & 205 & 98276144093 \\
\hline 166 & 5893180121 & 206 & 124221464119 \\
\hline 167 & 30827138509 & 207 & 49914935177 \\
\hline $168^{*}$ & 3842610773 & 208 & 121972158437 \\
\hline 169 & 22076314313 & 209 & 129300694603 \\
\hline 170 & 8605261447 & 210 & 82490815123 \\
\hline 171 & 12010745569 & 211 & 280974865361 \\
\hline 172 & 19724087267 & 212 & 264495345259 \\
\hline 173 & 11291401837 & 213 & 180265084403 \\
\hline 174 & 17002876643 & 214 & 219950168411 \\
\hline 175 & 16808773277 & 215 & 250964194171 \\
\hline 176 & 30750892801 & 216 & 87241770619 \\
\hline $177^{*}$ & 4302407359 & 217 & 127084569923 \\
\hline 178 & 24355072517 & 218 & 367459059871 \\
\hline 179 & 16792321339 & 219 & 101328529441 \\
\hline 180 & 20068818197 & 220 & 141846299801 \\
\hline 181 & 35877724601 & 221 & 417470554687 \\
\hline 182 & 25425617317 & 222 & 36172730063 \\
\hline 183 & 20108776097 & 223 & 190418076203 \\
\hline 184 & 51430518413 & 224 & 402872474743 \\
\hline 185 & 59942358571 & 225 & 63816175447 \\
\hline 186 & 20404137779 & 226 & 466855187471 \\
\hline 187 & 23064761663 & 227 & 202530831163 \\
\hline 188 & 16161669787 & $228 *$ & 25056082087 \\
\hline 189 & 38116957819 & 229 & 304040251469 \\
\hline 190 & 23323808741 & 230 & 131956235563 \\
\hline $191^{*}$ & 10726904659 & 231 & 400729567081 \\
\hline $192 *$ & 20678048297 & $232 *$ & 42652618343 \\
\hline 193 & 35238645587 & 233 & 565855695631 \\
\hline 194 & 156798792223 & $234^{*}$ & 127976334671 \\
\hline 195 & 53241805651 & 235 & 681753256133 \\
\hline 196 & 117215204531 & 236 & 865244709607 \\
\hline $197 *$ & 22367084959 & $237 *$ & 182226896239 \\
\hline
\end{tabular}


TABLE 1 (continued)

\begin{tabular}{lrlr}
\hline$r$ & $f(r)$ & $r$ & \multicolumn{1}{c}{$f(r)$} \\
\hline 238 & 725978934347 & $258^{*}$ & 416608695821 \\
239 & 367766547571 & 259 & 2296497058133 \\
240 & 482423533897 & 260 & 2336167262449 \\
241 & 1051602787181 & 261 & 1214820695701 \\
242 & 767644374817 & 262 & 2256065636039 \\
$243^{*}$ & 241160624143 & 263 & 1620505682371 \\
244 & 1275363152099 & 264 & 1529741785139 \\
$245^{*}$ & 297501075799 & 265 & 2205492372371 \\
246 & 910361180689 & $266^{*}$ & 461690510011 \\
247 & 804541404419 & $267^{*}$ & 614487453523 \\
248 & 880318998907 & 269 & 2122536905311 \\
249 & 428315806823 & $270^{*}$ & 738832927927 \\
$250 *$ & 303371455241 & 273 & 2164206784721 \\
251 & 1258535916601 & 275 & 2496646209271 \\
252 & 747431049203 & 276 & 2210401546601 \\
253 & 1339347750707 & 281 & 2081209441279 \\
254 & 1841086484491 & 282 & 1480064231153 \\
255 & 2209016910131 & $291 *$ & 1346294310749 \\
256 & 1999066711391 & $294^{*}$ & 1408695493609 \\
$257^{*}$ & 304599508537 & $301^{*}$ & 1968188556461 \\
\hline
\end{tabular}

The heuristic argument of Cadwell [3] suggests that $\phi(r) \simeq \frac{1}{4}$, but our computations indicate that $\phi(r)$ is usually in $\left(\frac{1}{2}, 1\right)$. Table 2 gives those values of $r$ for which $f(r) \leqq$ $2.6 \times 10^{12}$ and either $\phi(r) \leqq \frac{1}{2}$ or $\phi(r) \geqq 1$.

In number-theoretic computations it is often necessary to store blocks of consecutive primes. To save storage space, it may be convenient to store the semidifferences $\delta_{i}=\left(p_{i+1}-p_{i}\right) / 2$. Our computations show that $\delta_{i}<256$, so only eight bits of storage are required for each $\delta_{i}$, provided $p_{i} \leqq 3 \times 10^{11}$.

The largest gap found* was one of 601 composite numbers following the prime 1968188556461. From Brent [2], Jones, Lal and Blundon [9], and Weintraub [14], it is clear that strings of more than 400 consecutive composites between primes less than $10^{17}$ are extremely rare. For example, between $10^{16}$ and $10^{16}+10^{8}$ there are 2714904 primes, but only 15 gaps of more than 400 consecutive composites (the largest gap being of 531 composites).

2. Computation of Prime Gaps. To compute Table 1, integers up to $2.6 \times 10^{12}$ were sieved for primes in blocks of length $16336320=2^{6} \cdot 3 \cdot 5 \cdot 7 \cdot 11 \cdot 13 \cdot 17$. A one-bit flag represented each odd number in the block, so 1021020 bytes (each eight bits) of storage were required for the sieve. After setting all bits to zero, the bits corresponding to numbers divisible by $3,5,7,11, \cdots$ were set to one. (This required the primes up to

* Added in proof. In extending the search to $2.686 \times 10^{12}$ we found $f(272)=2652427555639$, $f(326)=2614941710599$, and $\phi(326)=0.4719$. 
TABLE 2

Values of $\phi(r)$ Lying Outside the Interval $\left(\frac{1}{2}, 1\right)$

\begin{tabular}{rrrr}
\hline$r$ & $\phi(r)$ & $r$ & $\phi(r)$ \\
\hline 1 & -0.4553 & 58 & 1.0120 \\
2 & -0.0390 & 74 & 0.4700 \\
3 & 0.3829 & 79 & 1.0159 \\
7 & 0.3735 & 83 & 1.0484 \\
8 & 1.2669 & 100 & 1.0585 \\
17 & 0.3856 & 105 & 0.4415 \\
19 & 1.1447 & 114 & 1.0155 \\
23 & 1.1820 & 167 & 1.0112 \\
36 & 0.4371 & 194 & 1.0200 \\
37 & 1.0010 & 228 & 0.4231 \\
56 & 0.4745 & 232 & 0.4781 \\
\hline
\end{tabular}

$\left(2.6 \times 10^{12}\right)^{1 / 2}$, which had been precomputed and stored as described in Section 1.) The remaining zero bits corresponded to primes, and these were efficiently scanned for large gaps by an algorithm like the first algorithm of [10].

The computations were performed on an IBM 360/91 computer at the IBM T. J. Watson Research Center, and various machine-dependent coding tricks were used to speed up the sieving and scanning. For example, with our choice of sieve size it was possible to set eight bits, corresponding to eight numbers divisible by $3,5,7,11,13$ or 17 , with just one MVI (move immediate) instruction. The program ran in 1194K bytes and required less than 7.2 seconds for each block of numbers near $2.6 \times 10^{12}$ (i.e., less than $4.4 \times 10^{-7}$ seconds per number).

The computed values of $f(r)$ were checked by a program which verified in a straightforward way that $f(r)$ and $f(r)+2 r$ were prime and all the intermediate integers were composite. Our results confirm those of Lander and Parkin [10], and it is unlikely that a machine or programming error could have resulted in a large gap being overlooked.

Computer Centre

Australian National University

Canberra, Australia

1. K. I. APpel \& J. B. Rosser, Table for Estimating Functions of Primes, IDA-CRD Technical Report Number 4, 1961, p. 102. (Reviewed in RMT 55, Math. Comp., v. 16, 1962, pp. 500-501.)

2. R. P. BRENT, Empirical Evidence for a Proposed Distribution of Small Prime Gaps, Technical Report CS 123, Computer Science Dept., Stanford Univ., Calif., 1969.

3. J. H. CaDWELl, "Large intervals between consecutive primes," Math. Comp., v. 25 , 1971, pp. 909-913.

4. H. CRAMÉR, "On the order of magnitude of the difference between consecutive prime numbers," Acta Arith., v. 2, 1937, pp. 23-46.

5. J. W. L. Glaisher, "On long successions of composite numbers," Messenger Math., v. 7, 1877, pp. 102, 171.

6. F. Gruenberger \& G. Armerding, Statistics on the First Six Million Prime Numbers, Paper P-2460, The RAND Corporation, Santa Monica, Calif., 1961, 145 pp. (Copy deposited in the UMT File and reviewed in Math. Comp., v. 19, 1965, pp. 503-505.) 
7. G. H. HaRdy \& J. E. Littlewood, "Some problems of 'partitio numerorum'; III: On the expression of a number as a sum of primes," Acta Math., v. 44, 1923, pp. 1-70.

8. S. M. JohNSON, "An elementary remark on maximal gaps between successive primes," Math. Comp., v. 19, 1965, pp. 675-676.

9. M. F. Jones, M. LAL \& W. J. Blundon, "Statistics on certain large primes," Math. Comp., v. 21, 1967, pp. 103-107. MR 36 \#3707.

10. L. J. LANDER \& T. R. PARKIN, "On first appearance of prime differences," Math. Comp., v. 21, 1967, pp. 483-488. MR 37 \#6237.

11. D. H. LEHMER, "Tables concerning the distribution of primes up to 37 millions," 1957. Copy deposited in the UMT File and reviewed in $M T A C$, v. 13, 1959, pp. 56-57. 393.

12. K. Pracher, Primzahlverteilung, Springer-Verlag, Berlin, 1957, pp. 154-164. MR 19,

13. D. Shanks, "On maximal gaps between successive primes," Math. Comp., v. 18, 1964, pp. 646-651. MR 29 \#4745.

14. S. WeInTRaub, "Distribution of primes between $10^{14}$ and $10^{14}+10^{8}, " 1971$. Copy deposited in the UMT File and reviewed in Math. Comp., v. 26, 1972, p. 596.

15. A. E. WESTERN, "Note on the magnitude of the difference between successive primes," J. London Math. Soc., v. 9, 1934, pp. 276-278. 